\title{
NUEVOS DATOS PALINOLÓGICOS DE LA FORMACIÓN KAKA (EOSERPUKHOVIANO) EN LA ENCAÑADA DE BEU, SUBANDINO NORTE DE BOLIVIA
}

\author{
ZULEMA FASOLO \\ Fundación Miguel Lillo, Inst. Paleontología, Área Geología, Miguel Lillo 251 (4000), San Miguel de Tucumán, \\ Argentina.fasolo@csnat.unt.edu.ar \\ MARÍA DEL MILAGRO VERGEL \\ CONICET, Universidad Nacional de Tucumán, Facultad de Ciencias Naturales e I.M.L., Miguel Lillo 205 (4000), \\ San Miguel de Tucumán, Argentina.vergelmar@tucbbs.com.ar \\ JAIME OLLER \\ PETROBRAS BOLIVIA, Av. San Martin 1700, Santa Cruz de la Sierra, Bolivia.joller@petrobras.com.bo
}

\section{CARLOS AZCUY}

CONICET, Universidad Nacional de Buenos Aires, Facultad de Ciencias Exactas y Naturales, Ciudad Universitaria, Pabellón 2 (1428), Buenos Aires, Argentina.azcuy@ciudad.com.ar

\begin{abstract}
RESUMO - São apresentadas novas informações sobre o conteúdo palinológico do topo da Formação Kaka, unidade superior do Grupo Retama, na Encañada de Beu, Subandino Norte da Bolívia. Identificaram-se 80 espécies de palinomorfos autóctones junto a outros espécimes do Devoniano, considerados como redepositados. A associação autóctone é basicamente composta por esporos triletes, uma espécie de pólen precolpado, duas espécies de grãos de pólen sacado e algas verdes (Botryococcus). Esta associação é tentativamente assinalada ao Serpunkoviano e correlacionada, apenas em parte, com a palinozona Cordylosporites magnidictyus, definida para o Viseano tardio da Bacia do Amazonas, no Brasil. É também sugerida uma correlação com a Formação Ambo, no Peru. As afinidades botânicas da maioria dos elementos constituintes da palinoflora indicam condições úmidas em um clima temperado.
\end{abstract}

Palavras-chave: Palinologia, Eocarbonífero, Formação Kaka, Bolívia.

\begin{abstract}
NEW PALYNOLOGYCAL RECORD IN THE KAKA FORMATION (EARLY SERPUKHOVIAN) AT ENCAÑADA DE BEU, SUBANDINO NORTE, BOLIVIA. New palynological information recovered from a well-preserved and diverse assemblage from the upper part of the Kaka Formation, upper unit of the Retama Group, at the Encañada de Beu, Northern Subandean Bolivia, is presented. Eighty autochthonous palynomorph species were identified together with reworked specimens from the Devonian s.l. The autochthonous assemblage is principally composed of trilete miospores, one praecolpate pollen species, two saccate pollen species, and green algae (Botryococcus). It is tentatively assigned to the Serpukhovian, and partly correlated with the Cordylosporites magnidictyus palynozone, defined in the Amazon basin (Brazil) and dated-late Viséan. The Bolivian assemblage is also correlated with the Ambo Formation assemblage from Peru. The major botanical affinities of the palynoflora suggest humid conditions in a temperate climate.
\end{abstract}

Key words: Palynology, Early Carboniferous, Kaka Formation, Bolivia.

\section{INTRODUCCIÓN}

El conocimiento de palinofloras carboníferas en América del Sur tuvo un sustancial avance en los últimos años, principalmente con los registros en secuencias de Brasil (Playford \& Dino, 2000; Melo \& Loboziak, 2000, 2003; di Pasquo et al., 2003a,b), Perú (Azcuy et al., 2002; Azcuy \& di Pasquo, 2005), y Argentina (di Pasquo et al., 2001; Rodríguez
Amenábar et al., 2003). En Bolivia sin embargo, sólo se han dado a conocer listas taxonómicas de palinomorfos obtenidas de informes inéditos de Yacimientos Petrolíferos Fiscales Bolivianos (Suárez-Soruco \& Lobo-Boneta, 1983; SuárezSoruco, 1989), y otros de mayor alcance como los llevados a cabo en el Norte del país, en la Encañada de Beu (Azcuy \& Ottone, 1987) y en el Noroeste, área del Lago Titicaca, Cuenca Madre de Dios (Vardová et. al., 1993, 1996). Estos dos últimos, 
corresponden a datos sobre la palinología de secciones estratigráficas en las cuales se presenta información referida al límite Devónico-Carbonífero.

La microflora dada a conocer por Azcuy \& Ottone (1987), a pesar de su carácter preliminar, brindó importantes datos sobre la antigüedad del nivel portador y permitió diferenciar materiales continentales y paleomicroplancton marino. El paleomicroplancton marino (acritarcos y prasinoficeas) además de varias esporas, fue interpretado como material redepositado del Devónico Tardío, mientras que la mayoría de sus especies continentales (esporas) fueron consideradas autóctonas con la depositación de las sedimentitas del Grupo Retama e interpretadas como del Carbonífero Temprano indiferenciado, aunque asignadas con dudas al Viseano.

En estos mismos niveles, Azcuy \& Suarez Soruco (1993) registraron megaflora con escasos especimenes de Nothorhacopteris kellaybelenensis, integrante principal de la Fitozona Nothorhacopteris kellaybelenensis Triphyllopteris boliviana (Iannuzzi et. al., 2003) cuya antigüedad se determina como Viseano tardío-Serpukhoviano temprano.

Dada la abundancia, diversidad y excelente preservación del material de la Encañada de Beu, se efectúa en este trabajo la revisión del material estudiado por Azcuy \& Ottone (1987) con el fin de actualizar y ampliar el registro taxonómico, correlacionar asociaciones semejantes descriptas recientemente para América del Sur, y eventualmente, ajustar los esquemas bioestratigráficos y paleogeográficos.

\section{MARCO GEOGRÁFICO Y GEOLÓGICO}

El área de estudio se halla ubicada en el departamento La Paz, en la unidad morfoestructural Subandino Norte de Bolivia (Figura 1). En la Llanura Beniana, la Encañada de Beu consiste en un área donde el río Beni corta una importante secuencia silicoclástica descubriendo una excelente exposición de Paleozoico que incluye el Grupo Retama (López-Murillo, 1967). Este grupo que representa parte del Ciclo Cordillerano (Ashgilliano superior-Serpukhoviano, sensu Suárez-Soruco, 2000), consiste en una unidad litoestratigráfica integrada por dos conjuntos sedimentarios asignados al Devónico más tardío y al Carbonífero Temprano respectivamente (SuárezSoruco, 2000). Diferentes autores hacen referencia a estos conjuntos sedimentarios con terminologías litoestratigráficas de distinta categoría. Algunos lo consideran una única formación integrada por dos miembros (Dávila et al., 1964), mientras que otros lo designan como un grupo con dos formaciones (Suárez-Soruco \& Díaz-Martínez, 1996). En este trabajo seguimos el criterio adoptado por los últimos autores mencionados que designan la secuencia como Grupo Retama, integrado por las Formaciones Toregua y Kaka (Figura 1).

La unidad inferior, Formación Toregua (López-Murillo, 1967), se dispone sobre areniscas del Devónico Superior de la Formación Tomachi y está mayormente constituida por areniscas cuarcíticas de grano fino a muy fino, bien estratificadas, en bancos de hasta $4 \mathrm{~m}$ de espesor, con delgadas intercalaciones de lutitas gris oscuras. Becar-
Montaño \& Toledo (1990) la interpretan como una sucesión de cuatro secuencias de progradación deltaica que suceden a la secuencia regresiva de línea de costa con la que culmina la serie devónica (Formaciones Tequeje y Tomachi), configurando un paleoambiente costero deltaico. La unidad superior, Formación Kaka (López-Murillo, 1967), se inicia en la base de la primer diamictita que registra la unidad, en una matriz areno-limosa, intercalada con niveles de pelitas y areniscas estratificadas; la sucesión se continúa con areniscas, limolitas y arcilitas en una secuencia grano y estrato decreciente. Según Suárez-Soruco (2000) representa una secuencia marina de plataforma somera, con marcada influencia glacimarina y evidentes rasgos de resedimentación. De los niveles pelíticos superiores de la Formación Kaka se obtuvo el material palinológico que analizamos en este trabajo, el cual corresponde además al nivel portador de Nothorhacopteris kellaybelenenensis, asignada al Carbonífero Temprano (Figura 1).

\section{MATERIALES Y MÉTODOS}

El material aquí analizado corresponde al tramo superior del Grupo Retama, en los niveles pelíticos superiores de la Formación Kaka (Figura 1). La extracción de los palinomorfos se realizó en el Laboratorio de Palinología del Departamento de Ciencias Geológicas de la Universidad de Buenos Aires, siguiendo métodos convencionales. Los materiales estudiados e ilustrados se hallan depositados en la Palinoteca del Laboratorio de Palinología con la sigla y número BAFC. Pl 506(1-30).

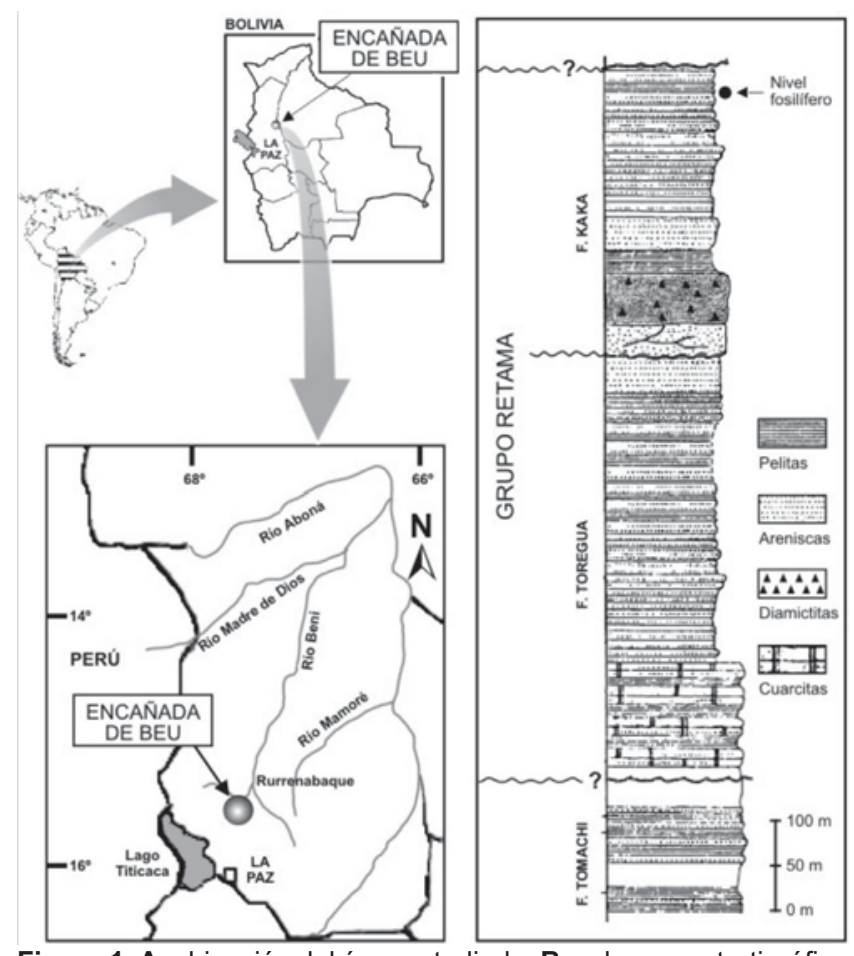

Figura 1. A, ubicación del área estudiada; B, columna estratigráfica del Grupo Retama y ubicación del nivel fosilífero.

Figure 1. A, location of the study area; $\mathbf{B}$, stratigraphic column of Retama Group and location of the fossiliferous horizon. 


\section{CARACTERÍSTICAS DE LA ASOCIACIÓN}

El análisis taxonómico permitió identificar una microflora muy diversificada con 120 formas, mayormente definidas a nivel específico. Entre ellas, de igual modo que fuera señalado por Azcuy \& Ottone (1987) se diferenciaron 2 grupos de palinomorfos, separados luego de su análisis taxonómico sobre la base de registros estratigráficos previos: a) un grupo de formas autóctonas compuesto de esporas y muy escasos granos de polen subordinados (ca. al $8 \%$ ); y b) un grupo alóctono integrado mayormente por formas marinas (acritarcas y prasinoficeas) y esporas continentales tales como Retispora lepidophyta, Samarisporites triangulatus, Emphanisporites rotatus, E. spinaeformis, Grandispora spp. de antigüedad devónica $s . l$. Esta asociación ocurre con una frecuencia de hasta un $40 \%$ y confirma los datos de retrabajo obtenidos de la sedimentología de la sección por Suarez Soruco (2000).

En el presente estudio analizamos exclusivamente el grupo de formas determinadas como autóctonas cuyos 80 taxa se listan en la Tabla 1; la misma incluye la cita previa de las especies encontradas nesta y en otras localidades de América del Sur. Algunos taxa se ilustran en las Figuras 2 a 4. La lista completa de los taxa constituye el Apéndice 1.

\section{EDAD DE LA PALINOFLORA AUTÓCTONA}

El significado estratigráfico de los taxa de la asociación autóctona, evaluado de acuerdo con su registro previo en otras asociaciones neopaleozoicas (Figura 5), indicaría para la microflora de Beni una antigüedad Carbonífero Temprano (Viseano tardío-Serpukhoviano). La asignación a esta edad se fundamenta en la presencia de formas comunes con asociaciones del Carbonífero Temprano mundial, como muestran los taxa seleccionados en la Figura 5. En ella se reconocen tres grupos de especies. El primero corresponde a especies con registro inicial en el Tournaisiano o Struniano pero que no sobrepasan el Viseano, e.g., Knoxisporites literatus, Cristatisporites echinatus, Kraeuselisporites mitratus, Retusotriletes crassus, Bascaudaspora submarginata, Verrucosisporites nitidus, Leiotriletes ornatus, Apiculeretusispora semisenta, Verrucosisporites congestus y $V$. depressus. El segundo grupo corresponde a especies con registro sólo en el Viseano, e.g., Verrucosisporites morulatus, Convolutispora insulosa, Grandispora debilis, las cuales sugieren una antigüedad viseana. Sin embargo, el tercer grupo corresponde a especies que si bien aparecen en el Viseano-Serpukhoviano y aún Tournaisiano, alcanzan la base del Carbonífero Tardío y aún un poco más, e.g., Cordylosporites magnidictyus, Waltzispora polita, Densosporites annulatus, Schopfipollenites ellipsoides, Cristatisporites indignabundus, Crassispora kosankei, Potonieisporites sp. y Limitisporites sp. Si bien la primera aparición de granos de polen monosacados es objeto de continuo debate, una vez que aparecen a nivel mundial con algunas variaciones diacrónicas. Al respecto, varios autores (Clayton et al., 1990;
Zhu, 1993; Clayton, 1995, 1996; Ouyang, 1996) concluyen que la ocurrencia esporádica de especies de granos de polen monosacados y bisacados, generalmente ocurren en el Namuriano Temprano o Serpukhoviano. Por lo arriba expuesto se asigna a la microflora una antigüedad Eoserpukhoviano. No obstante, la esporádica ocurrencia de Crassispora kosankei y algunos granos de polen en esta asociación deja abierta la posibilidad a futuras revisiones de los niveles, y a nuevas colecciones en el área.

\section{CORRELACIÓN CON ASOCIACIONES NEOPALEOZOICAS TEMPRANAS DE AMÉRICA DEL SUR}

La presencia de taxa del Carbonífero Tardío y/o del límite entre los subsistemas (Carbonífero Temprano y Tardío) reforzarian la asignación de esta asociación a una antigüedad Eoserpukhoviano. Por otra parte, la asociación de Beni estudiada por Azcuy \& Ottone (1987), fue originalmente comparada con la Palynozona Verrucosisporites sp. $85(=\mathrm{V}$. nitidus) - Reticulatisporites magnidictyus (= Cordylosporites magnidictyus) Suárez-Soruco \& Lobo Boneta (1983) y atribuida al Viseano s.l., que posteriormente fue reasignada al Viseano tardío-Serpukhoviano por su asociación con la Fitozona Nothorhacopteris kellaybelenesis - Triphyllopteris boliviana (Iannuzzi et. al., 2003).

De los datos mostrados en la Tabla 1, surge claramente que la asociación con mayor número de especies comunes (25) con la asociación aquí estudiada, es la del Pongo de Mainique, Perú (Formación Ambo), Cuenca Madre de Dios. La asociación de la Formación Ambo fue asignada al Viseano tardío, cabe resaltar la ausencia de granos de polen monosacados, y correlacionada con la palinozona Cordylosporites magnidictyus (Melo \& Loboziak, 2003) de la Cuenca Amazonas, Brazil (Azcuy \& di Pasquo, 2005).

Melo \& Loboziak (2003) proponen en la Cuenca Amazonas

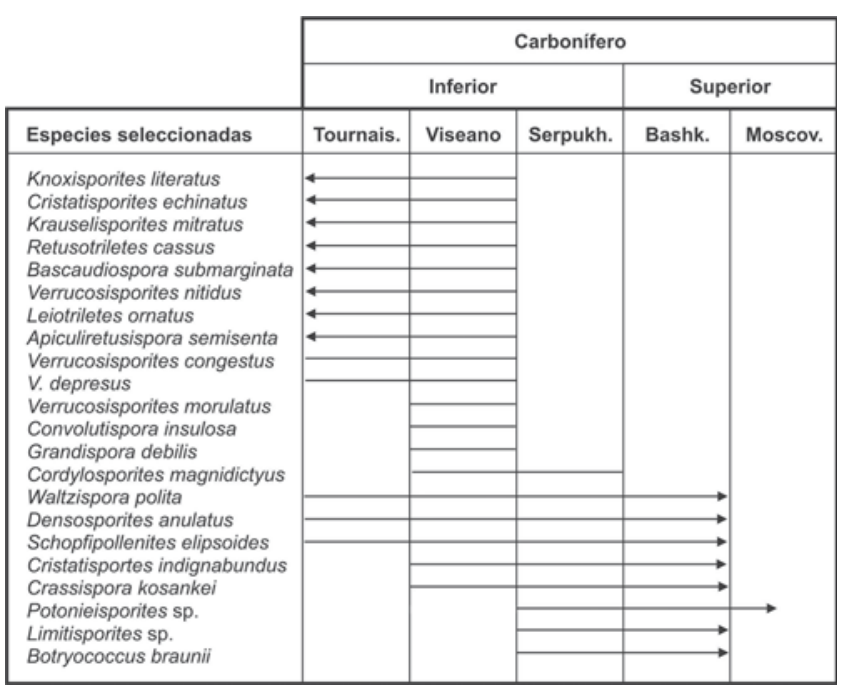

Figura 2. Distribución estratigráfica de especies seleccionadas. Figura 2. Stratigraphic distribution of selected species. 

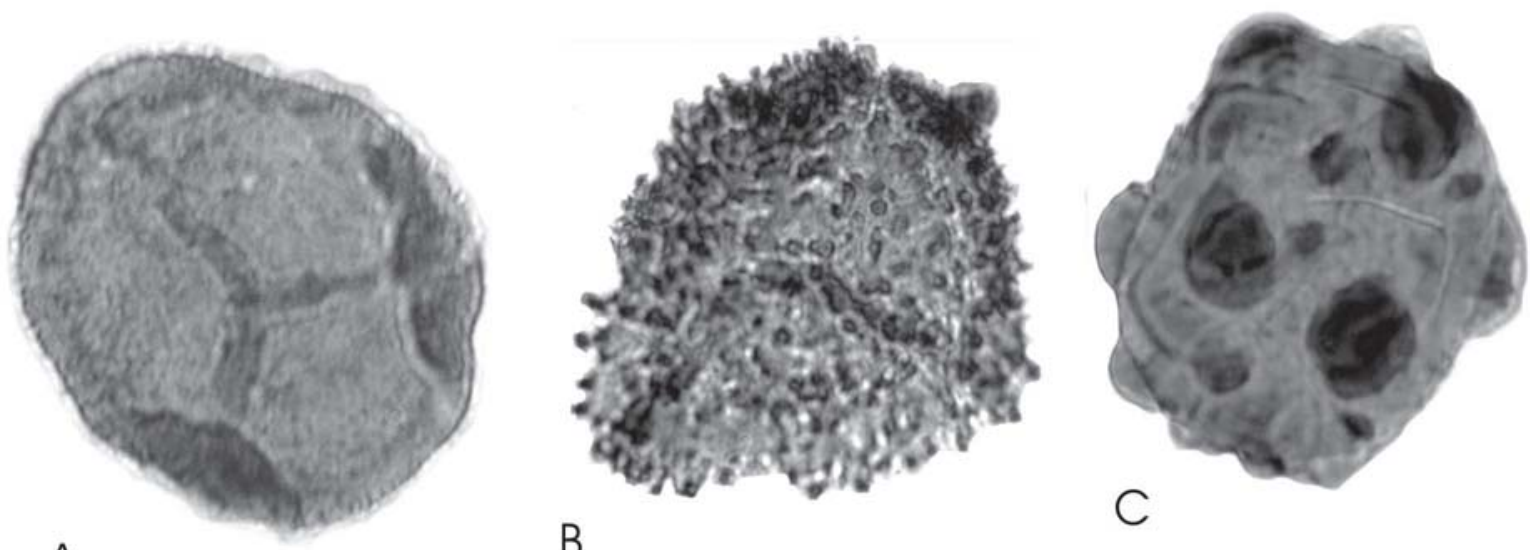

A

B
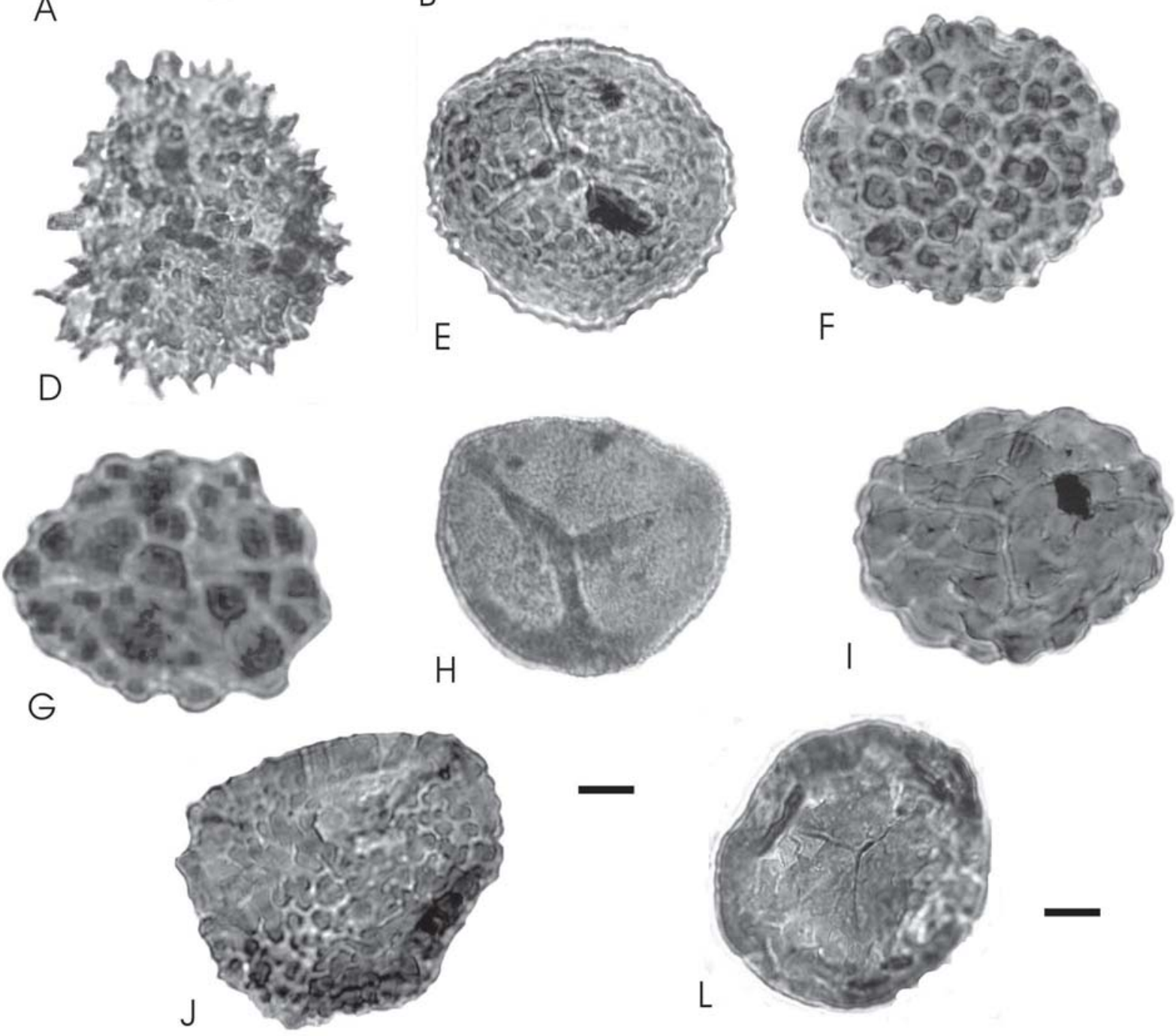

Figura 3. Asociación de esporas de la Formación Kaka (en paréntesis están el número de los preparados y la coordenada England Finder): A, Aneurospora gregsii (506/15, M35/2); B, Cristatisporites echinatus (506/28, 47/1); C, Verrucosisporites congestus (506/21, M56/4-N56/2); D, Cristatisporites indignabundus (506/18, G60/1); E, Verrucosisporites depressus (506/15, L54/2); F, Verrucosisporites morulatus (506/26, O32/1); G, Verrucosisporites nitidus (506/11, F65/1); H, Apiculiretusispora semisenta (506/15, M35/2); I, Convolutispora insulosa (506/17, M25/4); J, Bascaudaspora submarginata (506/28, D46/1); L, Retusotriletes crassus (506/18, S48/1). Todas x750, salvo las con barra de escala $(=7,5 \mu \mathrm{m})$.

Figure 3. Spores from Kaka Formation (in parenthesis are the slide number and the England Finder coordinates). All x750, excepting those with scale bar $(=7.5 \mu \mathrm{m})$. 

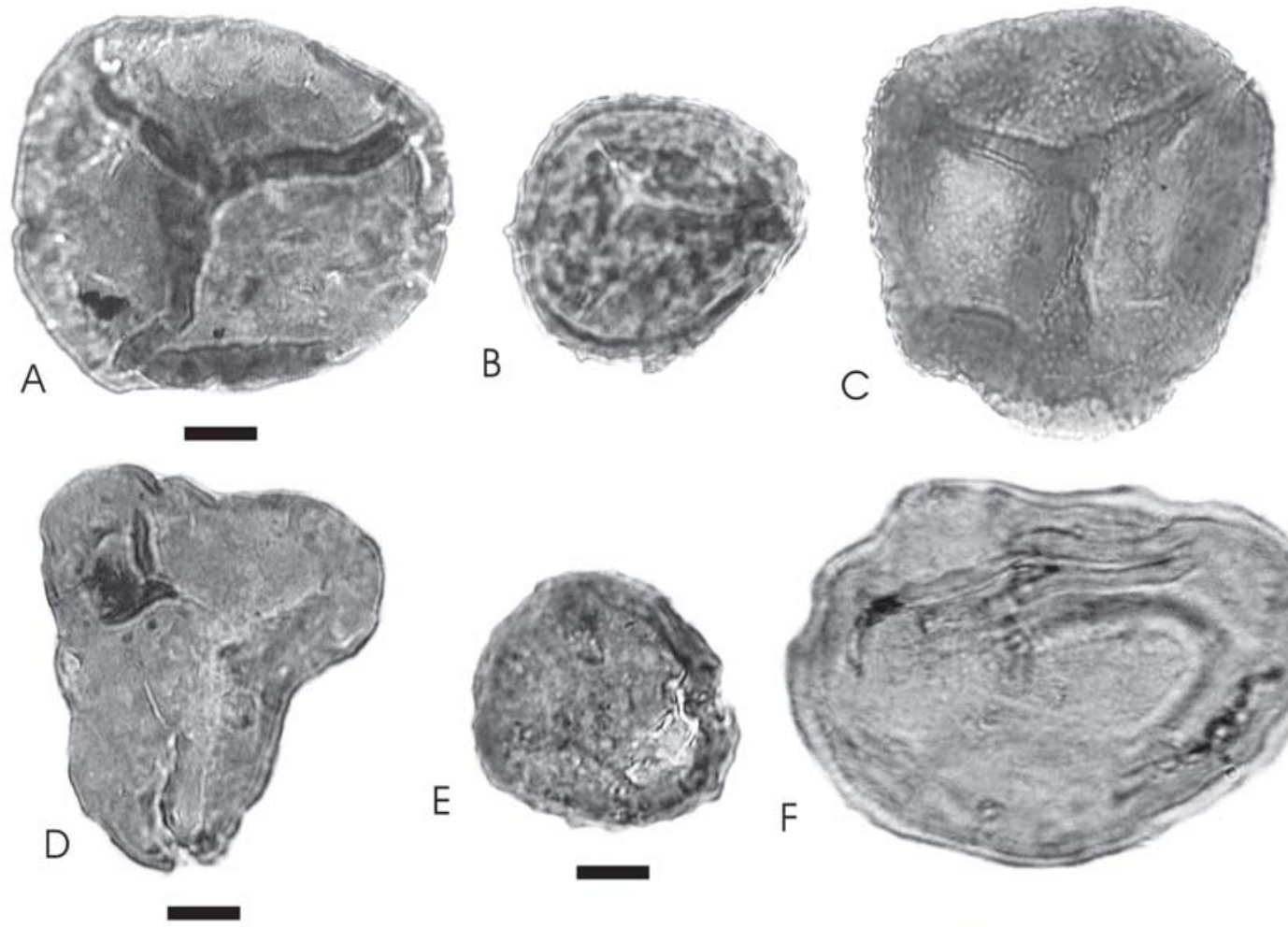

E
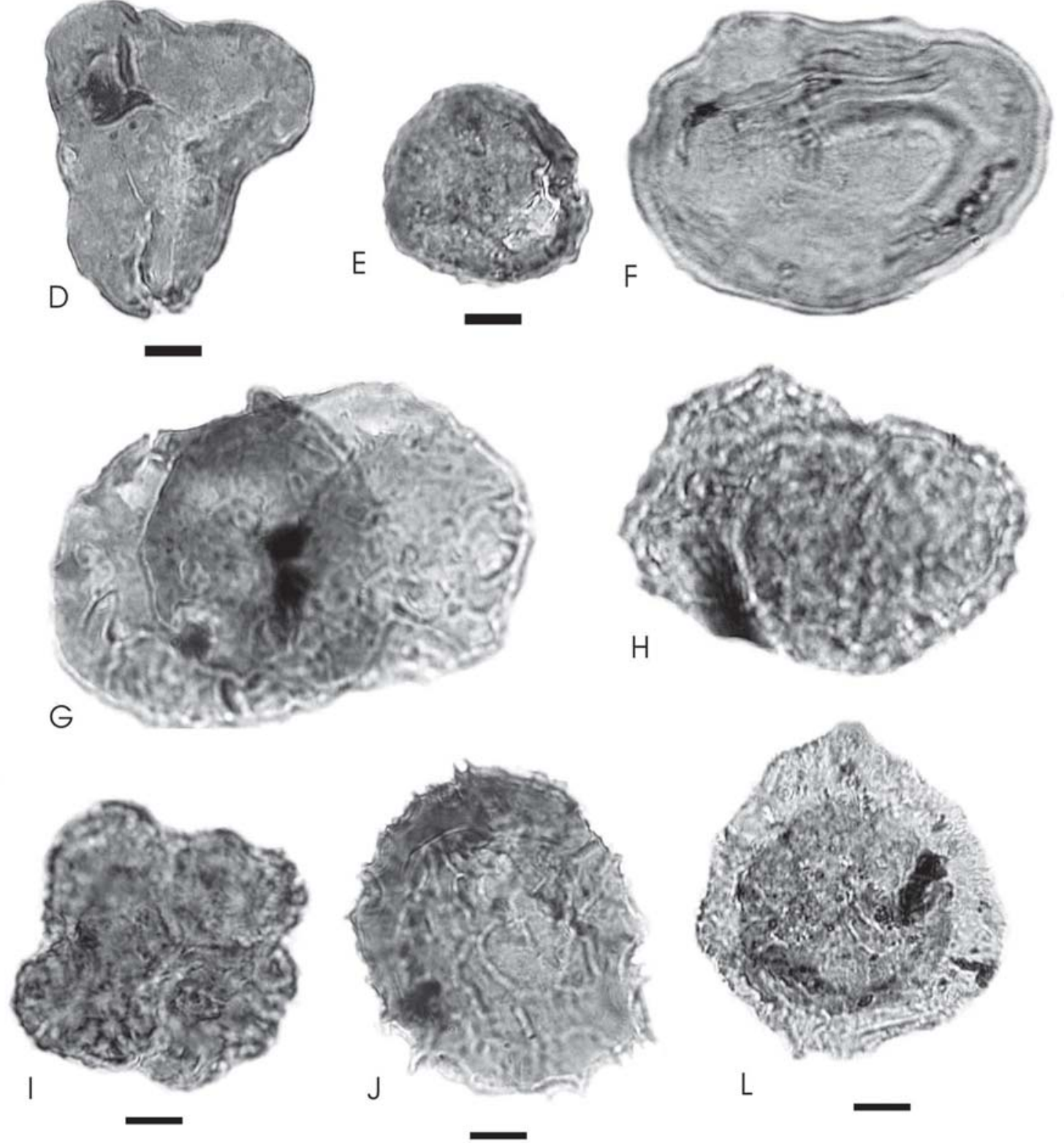

Figure 4. Asociación de esporas y granos de pollen de la Formación Kaka (en paréntesis están el número de los preparados y la coordenada England Finder): A, Leiotriletes ornatus (506/19, L54/2); B, noxisporites literatus (506/1, V21/4); C, Crassispora kosankei (506/18, Y28/2); D, Waltzispora polita (506/27, B39/3); E, Densosporites anulatus (506/11, L37/3-M37/1); F, Schopfipollenites ellipsoides (506/1, H20/2); G, Potonieisporites sp. (506/18, O47/3); H, Limitisporites sp. (506/12, R61/1-3); I, Botryococcus braunii (506/16, V53/1); J, Cordylosporites magnidictyus (506/1, Y23/2); L, Grandispora debilis (506/21, T34/4). Todas x750, salvo A, D, E, I, J y L x1000 y las con barra de escala $(=10 \mu \mathrm{m})$.

Figure 4. Miospores and pollen grains from Kaka Formation (in parenthesis are the slide number and the England Finder coordinates). All $x 750$, excepting $A, D, E, I, J$ and $L \times 1000$ and those with scale bar $(=10 \mu \mathrm{m})$. 


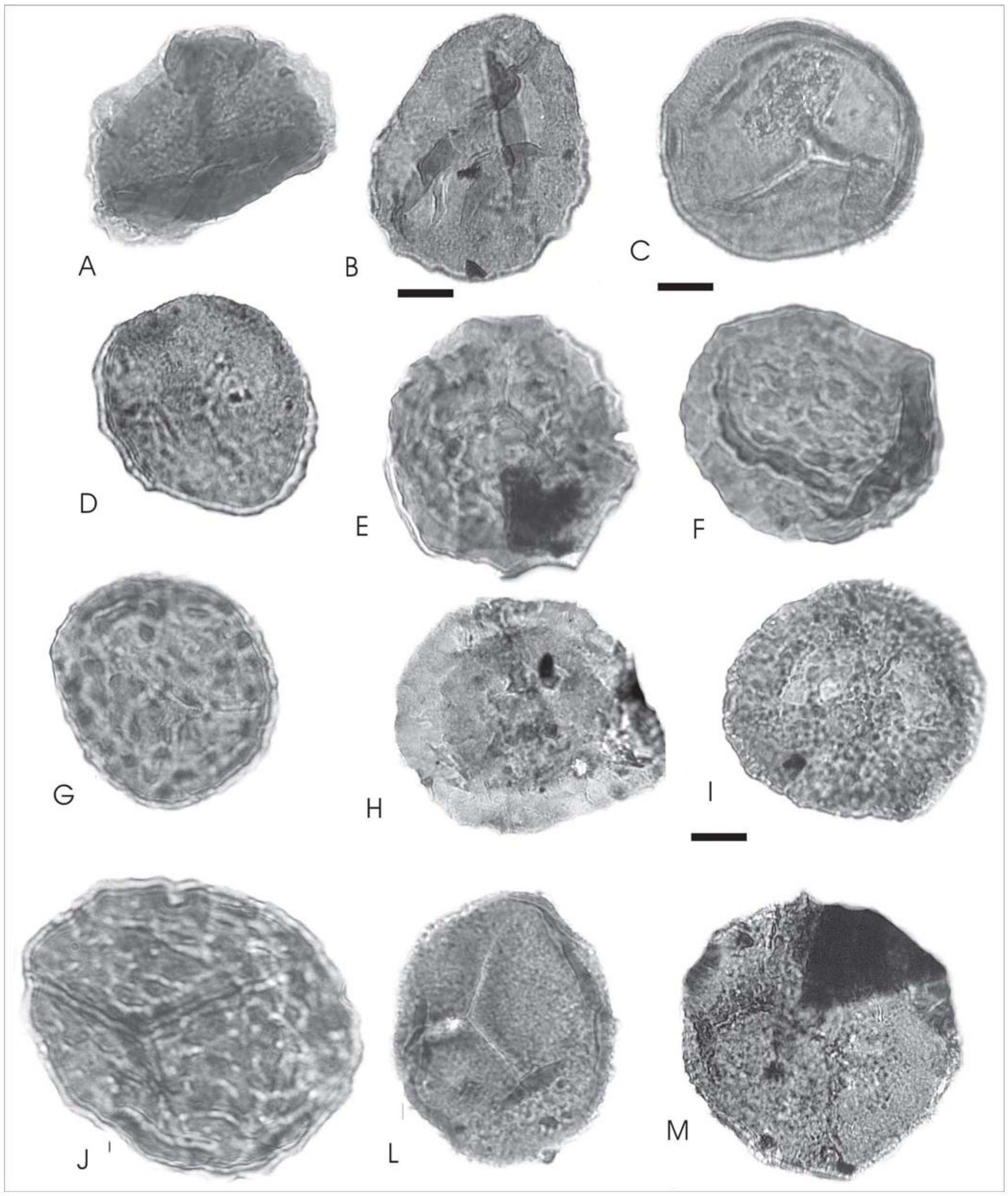

Figura 5. Asociación de esporas de la Formación Kaka (en paréntesis están el número de los preparados y la coordenada England Finder): A, Velamisporites sp. (506/12, W43/1); B, Granulatisporites parvus (506/18, U43); C, Cyclogranisporites pisticus (506/16, T57/ 3); D, Cyclogranisporites australis (506/19, R40/3); E, Grumosisporites rufus (506/24, Z33); F, Grumosisporites varioreticulatus (506/ 15, Y50/3); G, Grumosisporites verrucosus (506/15, R53); H, Endoculeospora gradzinskii (506/1, F25); I, Foveosporites hortonensis (506/18, P43/3-Q43/1); J, Foveosporites pellucidus (506/19, G59); L, Granasporites medius (506/3, X27); M, Spelaeotriletes arenaceus (506/7, P48/4). Todas $\times 750$, salvo B, C y I x1000 y las con barra de escala $(=10 \mu \mathrm{m})$.

Figure 5. Spores from Kaka Formation (in parenthesis are the slide number and the England Finder coordinates). All x750, excepting B, C and I $\mathrm{x} 1000$ and those with scale bar $(=10 \mu \mathrm{m})$. 
Tabla 1. Especies autóctonas registradas en la Formación Kaka (presente trabajo) y su distribución geográfica en América del Sur: Formación Retama, en la misma localidad (Azcuy \& Ottone, 1987); Formación Saipurú (Suárez-Soruco \& Lobo Boneta, 1983); Formación Faro (Melo \& Loboziak, 2003); Formación Poti (Melo \& Loboziak, 2000); Formación Ambo (Azcuy \& di Pasquo, 2004); Formación Cortaderas (Césari \& Limarino, 1992); Formación Malimán (Césari \& Limarino, 1995; Rodríguez Amenábar et al., 2003); Formación Del Ratón (Sessarego \& Césari, 1989); Formación Tupambi (di Pasquo, 2002).

Table 1. Autochthonous palynomorph species from the Kaka Formation (this paper) and their geographic distribution in South America: Retama Formation, at the same locality (Azcuy \& Ottone, 1987); Saipurú Formation (Suárez-Soruco \& Lobo Boneta, 1983); Faro Formation (Melo \& Loboziak, 2003); Poti Formation (Melo \& Loboziak, 2000); Ambo Formation (Azcuy \& di Pasquo, 2004); Cortaderas Formation: Río Blanco Basin, Argentina (Césari \& Limarino, 1992); Malimán Formation (Césari \& Limarino, 1995, Rodríguez Amenábar et al. 2003); Del Ratón Formation (Sessarego \& Césari, 1989); Tupambi Formation (di Pasquo, 2002).

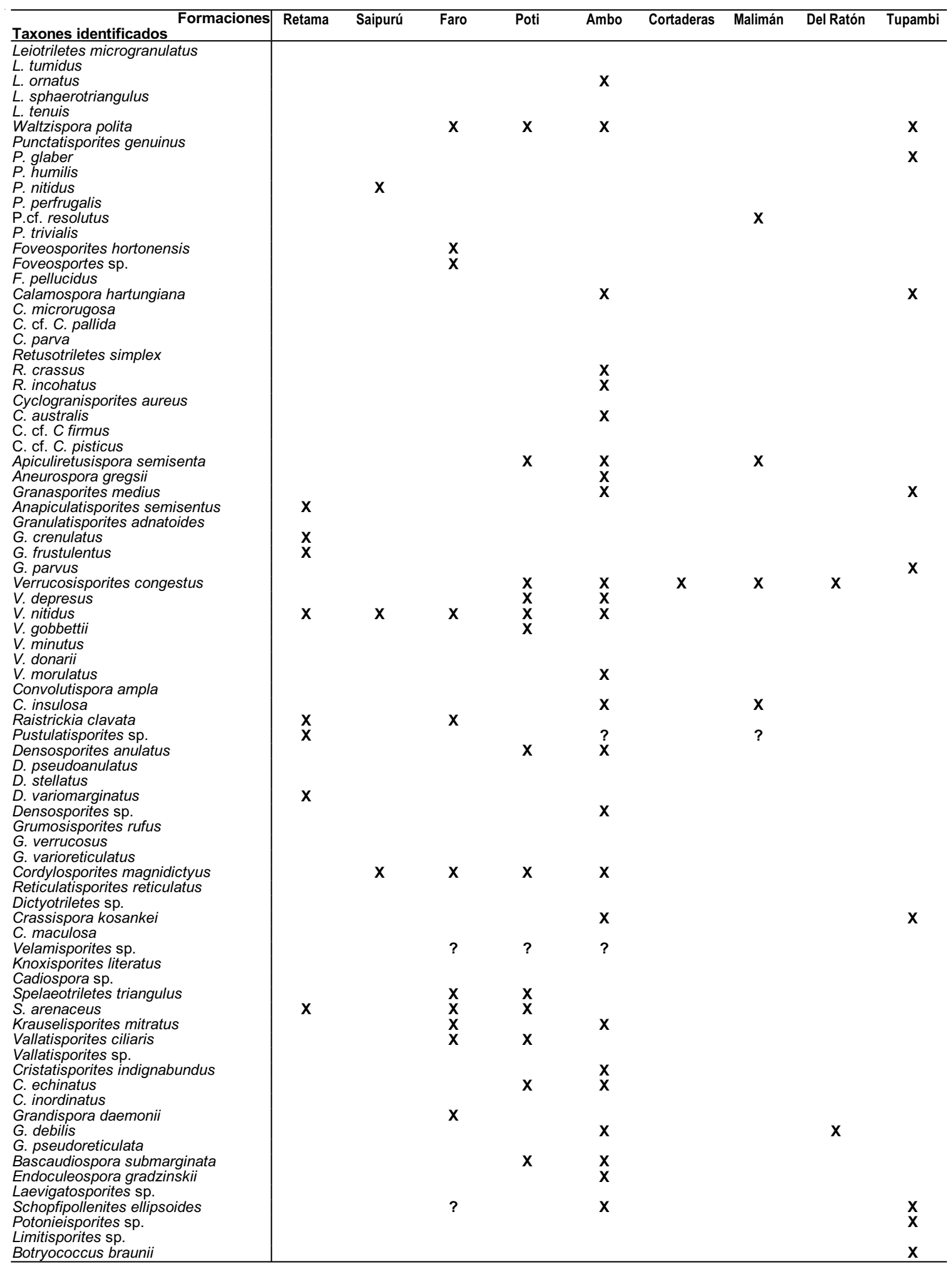


un esquema bioestratigráfico palinológico para el DevónicoCarbonífero Temprano. Para el post-Tournaisiano dichos autores establecieron la Palinozona Cordylosporites magnidictyus (Mag) entre cuyas formas 11 son comunes con la asociación de Beni, conforme Tabla 1, además de formas de rango más extenso que si bien persisten desde el Struniano, no proliferan más allá de la zona Mag. En tanto que otros componentes de la asociación como Waltzispora polita, Spelaeotriletes triangulus, S. arenaceus, Granulatisporites spp., están también presentes en la Cuenca Amazonas en asociaciones más jóvenes (Palinozona Spelaeotriletes triangulus, Tri) de probable edad Namuriano tardíoWestfaliano temprano. Así, el contar con varias especies en común, incluyendo las formas restringidas a la zona, se sugiere la correlación parcial de la asociación de Beni con la Palinozona Cordylosporites magnidictyus asignada al Viseano tardio.

Con respecto a la palinozona Spelaeotriletes triangulus (Tri) ciertos taxa son comunes con la asociación de Beni (Waltzispora polita, Spelaeotriletes triangulus, Potonieisporites sp., Limitisporites sp.) no obstante, la presencia de un alto predominio de granos de polen (monosacados, bisacados y estriados) en esa biozona aleja su correlación. También puede correlacionarse la microflora proveniente de la Formación Poti de la Cuenca Parnaíba (Melo \& Loboziak, 2000). Tienen 13 especies en común (Tabla 1), asignada al más tardío Viseano.

En Argentina las microfloras carboníferas tempranas fueron registradas en Cuencas del Oeste (Calingasta-Uspallata y Río Blanco) en afloramientos de las Formaciones Del Ratón, Malimán y Cortaderas. Estas microfloras son incluidas por Césari \& Gutiérrez (2000) en la biozona Cordylosporites Verrucosisporites propuesta por dichos autores para la región centro oeste de Argentina y asignadas al Tournaisiano tardíoViseano temprano. La correspondencia con la microflora de Beni es mínima con escasas especies en común como Verrucosisporites congestus citada para la Formación Cortaderas de la Cuenca Río Blanco (Césari \& Limarino, 1992) y Apiculiretusispora semisenta, Convolutispora insulosa en la Formación Malimán de la misma cuenca (Césari \& Limarino, 1995). Por otra parte, la revisión de la asociación palinológica de la Formación Malimán comenzada por Rodríguez Amenábar et al. (2003), permitió reconocer en niveles basales y medios de esa unidad Apiculiretusispora multiseta, A. semisenta, Convolutispora insulosa y Grandispora debilis. Para la Cuenca Calingasta-Uspallata, Verrucosisporites congestus y Grandispora debilis, registradas por Sessarego \& Césari (1989) en la Formación Del Ratón, completan las formas comunes con Bolivia. Tal vez la mínima correspondencia entre las asociaciones de Bolivia y Argentina pueda obedecer a las distintas posiciones paleolatitudinales entre ambas regiones durante la depositación de sus microfloras (Azcuy \& di Pasquo, 2004).

Al Norte de Argentina, muestras de superficie y subsuelo de la sección media a superior de la Formación Tupambi han brindado una microflora compuesta principalmente por esporas triletes y granos de polen monosacados que permitieron proponer la palinozona Crassispora kosankei Cystoptychus azcuyi (di Pasquo, 2002), asignada al Namuriano tardío. Esta palinozona registra algunos elementos comunes con la microflora de Beni como Schopfipollenites ellipsoides, Waltzispora polita, Punctatisporites glaber, Granasporites medius, Crassispora kosankei, Calamospora hartungiana y Botryococcus braunii. Estos elementos se asignan a una edad cercana al límite Carbonífero Temprano-Tardío. No obstante, existe una clara diferencia entre ambas microfloras por la ausencia, en la asociación de Beni, de los marcadores de la palinozona del norte argentino. La ausencia en la asociación de Bolivia de las especies características de la biozona, la falta de abundancia relativa de granos de polen monosacados y de otras especies características de palinofloras más modernas justifican su separación en el tiempo.

\section{SIGNIFICADO PALEOAMBIENTAL}

La palinoflora autóctona de la Formación Kaka (Tabla 1) está mayoritariamente compuesta de esporas cuya afinidad botánica muestra un fuerte dominio (68\%) de especies relacionadas a diferentes grupos de Pteridofitas conforme Balme (1995) (Figuras 2-4). Las Filicofitas (Zygopteridales, Botryopteridales, Cladoxylales, Marattiales, Filicales) que corresponden al 34\%, están representadas por géneros como: Leiotriletes, Punctatisporites, Cyclogranisporites, Granulatisporites, Verrucosisporites, Convolutispora, Raistrickia, Grumosisporites, Reticulatisporites, Dictyotriletes, Knoxisporites, Grandispora. También hay alta frecuencia de Licofitas (28\%) representados por: Foveosporites, Retusotriletes, Aneurospora, Granasporites, Anapiculatisporites, Densosporites, Crassispora, Kraeuselisporites, Vallatisporites y Cristatisporites. Esporas atribuidas a Esfenofitas como Calamospora, Auroraspora, Laevigatosporites se hallan subordinadas (6\%).

Los granos de polen precolpados de Schopfipollenites cuya afinidad botánica se indica como perteneciente a las Pteridospermofitas Medullosaceae en las palinofloras del Hemisferio Norte, y los esporádicos granos de polen Potonieisporites y Limitisporites atribuidos a Cordaitales y/ o Confierales ocurren en una frecuencia de aproximadamente un $8 \%$. Esto podría indicar que además de las plantas Pteridofitas, crecían en zonas no muy alejadas de la cuenca especies pertenecientes a grupos gimnospérmicos.

Las afinidades botánicas sugieren condiciones ambientales húmedas debido al dominio de elementos vinculados a grupos de plantas con requerimientos higrohidrófilos. La presencia de algas verdes Botryococcaceae se interpreta mayormente como vinculada a cuerpos de agua dulce de ambiente continental (Batten \& Grenfell, 1996).

Por otro lado, la megaflora de Nothorhacopteris asociada, fue considerada en Bolivia integrante del Cinturón Florístico Intra-Gondwana o Reino Paraca (Alleman \& Pfefferkorn, 1988; Iannuzzi et al., 2003) cuyas floras de climas templados y húmedos se desarrollaron en una amplia franja que se extendió 
desde el Oeste de América del Sur hasta el Este de Australia durante el Carbonífero Temprano (Viseano tardíoSerpukhoviano) (Iannuzzi \& Pfefferkorn, 2002)

\section{CONCLUSIONES}

Se amplia a 80 el número de especies palinológicas autóctonas en la Formación Kaka, en la Encañada de Beu, Bolivia, o que hay permitido asignar una antigüedad Eoserpukhoviano a la asociación. Además, se correlaciona esa microflora con la Palinozona Cordylosporites magnidictyus (en parte) propuesta en la Formación Faro, Cuenca Amazonas, Brasil y con las asociaciones de la Formación Ambo, Cuenca Madre de Dios, Perú y con la de la Formación Poti, Cuenca Parnaíba, Brasil, hallándose una amplia correspondencia entre ellas. Por otro lado, la correlación con microfloras del Carbonífero Temprano de Argentina es por ahora pobre, posiblemente a causa de una posición paleolatitudinal diferente durante el período de vida de las plantas productoras de las microfloras. Por fin, se infieren, sobre la base de los requerimientos de sus plantas parentales, condiciones húmedas durante el depósito de la microflora y, posiblemente clima templado por la posición paleolatitudinal que habría alcanzado esa región durante el Carbonífero Temprano.

\section{BIBLIOGRAFÍA}

Alleman, V. \& Pfefferkorn, H., 1988. Licópodos de Paracas: Significación geológica y paleo-climatológica. Boletín de la Sociedad Geológica del Perú, 78:131-136.

Azcuy, C.L. \& di Pasquo, M.M. 2004. Palinología de las formaciones Ambo y Tarma (Carbonífero) de Perú y su comparación con otras microfloras coetáneas. In: REUNIÃO DE PALEOBOTÂNICOS E PALINÓLOGOS, 11, 2004. Boletim de Resumos, Gramado, p. 14.

Azcuy, C.L. \& di Pasquo, M.M. 2005. Early Carboniferous palynomorphs from the Ambo Formation, Pongo de Mainique, Peru. Review of Palaeobotany and Palynology, 134:153-184.

Azcuy, C.L. \& Ottone, G. 1987. Datos palinológicos de la Formación Retama en la Encañada de Beu, Río Alto Beni (Bolivia). In: CONGRESO LATINOAMERICANO DE PALEONTOLOGÍA, 4, 1987. Actas, Santa Cruz de la Sierra, 1:235-249.

Azcuy, C.L. \& Suárez Soruco, R. 1993. Nothorhacopteris kellaybelenensis, una nueva especie del Carbonífero inferior de Bolivia. Revista Técnica de Yacimientos Petroliferos Fiscales Bolivianos, 13-14:173-179.

Azcuy, C.L.; di Pasquo, M.M. \& Valdivia Ampuero, H. 2002. Late Carboniferous miospores from the Tarma Formation, Pongo de Mainique, Perú. Review of Palaeobotany and Palynology, 118:1-28.

Balme, B.E., 1995. Fossil in situ spores and pollen grains: an anotate catalogue. Review of Palaeobotany and Palynology, 87:81-323.

Batten, D.J. \& Grenfell, H.R. 1996. Botryococcus. In: J. Jansonius \& D.C. Mc Gregor (eds.) Palynology: principles and applications, American Association Stratigraphic and Palynologists Foundation, v. 1, p. 205-214.

Becar-Montaño, G. \& Toledo, H., 1990. Estratigrafía de detalle. Area septentrional del Subandino Norte. Santa Cruz de la Sierra, 45 p. (Informe interno YPFB - GXG-3294).

Césari, S.N. \& Gutiérrez, P.R. 2000. Palynostratigraphy of Upper
Paleozoic sequences in central-western Argentina. Palynology, 24:113-146.

Césari, S.N. \& Limarino, C.O. 1992. Palinomorfos Eocarboníferos en la Formación Cortaderas, provincia de San Juan, Argentina. In: SIMPOSIO ARGENTINO DE PALEOBOTÁNICA Y PALINOLOGÍA, 8, 1992. Publicación Especial, Buenos Aires, APA, 2:45-48.

Césari, S.N. \& Limarino, C.O., 1995. Primer registro palinológico de la Formación Malimán (Carbonífero Inferior), Cuenca Río Blanco, Argentina. In: CONGRESO ARGENTINO DE PALEONTOLOGÍA Y BIOESTRATIGRAFÍA, 6, 1994. Actas, Trelew, p. 77-83.

Clayton, G., 1995. Carboniferous miospore and pollen assemblages from the Kingdom of Saudi Arabia. Review of Palaeobotany and Palynology, 89:115-123.

Clayton, G. 1996. Mississippian miospores. In: J. Jansonius \& D.C. McGregor (eds.) Palynology: principles and applications, American Association Stratigraphic and Palynologists Foundation, v. 2, p. 589-596.

Clayton, G.; Loboziak, S.; Streel, M.; Turnau, E. \& Utting, J. 1990. Palynological events in the Mississippian (Lower Carboniferous) of Europa, North Africa and North America. Courier Forschungsinstitut Senckenberg, 130:79-84.

Dávila, J.; Vargas, C. \& Ponce de León, V. 1964. Informe sobre la geología del bloque andino noroccidental y la faja subandina del norte, La Paz, 45 p. (Informe interno YPFB - GXG-1000).

di Pasquo, M.M., 2002. The Crassispora kosankei - Cystoptychus azcuyi palynozone of the Upper Carboniferous Tupambi Formation, Tarija Basin, northern Argentina. Review of Palaeobotany and Palynology, 118:47-76.

di Pasquo, M.M; Azcuy, C.L. \& Starck, D. 2001. Palinología de la Formación San Telmo en la sierra San Antonio, provincia de Salta, Argentina. Ameghiniana, 38:85-98.

di Pasquo, M.M.; Azcuy C.L. \& Souza, P. 2003a. Palinología del Carbonífero Superior del Subgrupo Itararé en Itaporanga, Cuenca Paraná, Estado de San Pablo, Brasil. Parte 1: sistemática de esporas y paleofitoplancton. Ameghiniana, 40:277-296

di Pasquo, M.M.; Azcuy C.L. \& Souza, P. 2003b. Palinología del Carbonífero Superior del Subgrupo Itararé en Itaporanga, Cuenca Paraná, Estado de San Pablo, Brasil. Parte 2: sistemática de polen y significado estratigráfico. Ameghiniana, 40:297-313.

Iannuzzi, R. \& Pfefferkorn, H. 2002. A Pre-Glacial, WarmTemperate Floral Belt in Gondwana (Late Viséan, Early Carboniferous). Palaios, 17:571-590.

Iannuzzi, R.; Azcuy, C.L. \& Suárez-Soruco, R. 2003. Fitozona Nothorhacopteris kellaybelenensis-Triphyllopteris boliviana, una nueva unidad bioestratigráfica para el Carbonífero Temprano de Bolivia. Revista Técnica de Yacimientos Petroliferos Fiscales Bolivianos, 21:125-131.

López-Murillo, H. 1967. Acerca de la geología de las sierras subandinas del norte. Boletín del Instituto Boliviano del Petróleo, 7(2):14-27.

Melo, J.H.G, Loboziak, S. 2000. Viséan miospore biostratigraphy and correlation of the Poti Formation (Parnaíba Basin, northern Brazil). Review of Palaeobotany and Palynology, 112:147-165.

Melo, J.H.G, Loboziak, S. 2003. Devonian-Early Carboniferous miospore biostratigraphy of the Amazon Basin, Northern Brazil. Review of Palaeobotany and Palynology, 124:131-202.

Ouyang, S. 1996. On the first appearance of some gymnospermous pollen and GSPD assemblages in the sub-Angara, Euramerian and Cathaysia provinces. The Palaeobotanist, 45:20-32.

Playford, G. \& Dino, R. 2000. Palynostratigraphy of Upper 
Paleozoic strata (Tapajós Group), Amazonas Basin, Brazil: Part Two. Palaeontographica, Abt. B, 255:87-145.

Rodríguez Amenábar, C.; di Pasquo, M.M. \& Carrizo, H.A. 2003. Datos palinológicos del límite Devónico-Carbonífero en la quebrada Cortaderas, provincia de San Juan, Argentina. Ameghiniana, Supl., 40(4):36-37.

Sessarego, H.L. \& Césari, S.N. 1989. An early Carboniferous flora from Argentina. Biostratigraphic implications. Review of Palaeobotany and Palynology, 57:247-264.

Suárez-Soruco, R. 1989. El ciclo Cordillerano (Silúrico-Carbonífero Inferior) en Bolivia y su relación con países limítrofes. Revista Técnica de Yacimientos Petroliferos Fiscales Bolivianos, 10(34):233-243.

Suárez-Soruco, R. 2000. Compendio de Geología de Bolivia. Revista Técnica de Yacimientos Petrolíferos Fiscales Bolivianos, 18(12): $1-213$.

Suárez-Soruco, R. \& Díaz-Martínez, E. 1996. Léxico Estratigráfico de Bolivia. Revista Técnica de Yacimientos Petroliferos Fiscales Bolivianos, 17(1-2):3-227.

Suárez-Soruco, R. \& Lobo-Boneta, J. 1983. La fase compresiva Eohercínica en el sector oriental de la Cuenca Cordillerana de Bolivia. Revista Técnica de Yacimientos Petroliferos Fiscales Bolivianos, 9(1-4):189-202.

Vavrdová, M.; Isaacson, P.E.; Díaz-Martinez, E. \& Bek, J. 1993. Devonian-Carboniferous boundary at Lake Titikaka, Bolivia: preliminary palynological results. In: CONGRÈS INTERNATIONAL DE LA STRATIGRAPHIE ET GEOLOGIE DU CARBONIFÈRE ET PERMIEN, 12, 1991. Comptes Rendus, Buenos Aires, 1:187-200.

Vavrdová, M.; Bek, J.; Dufka, P. \& Isaacson, P.E. 1996. Palynology of the Devonian (Lochkovian to Tournaisian) sequence, Madre de Díos, northern Bolivia. Véstnik Ceského geologického ústavu, 71(4):333-349.

Zhu, H.G. 1993. A revised palynological sub-division of the Namurian of Jing-yuan, northwest China. Review of Palaeobotany and Palynology, 77:273-300.

Received in January, 2006; accepted in March 2006.

Apéndice 1. Lista alfabética de taxa identificados.

Appendix 1. Alphabetic list of identified taxa.

Anapiculatisporites semisentus Playford 1971

Aneurospora gregsii (McGregor) Streel in Becker, Bless, Streel

\& Thorez 1974

Apiculiretusispora semisenta (Playford) Massa, Coquel,

Loboziak \& Tagourdeau-Lantz 1980

Bascaudaspora submarginata (Playford) Higgs, Clayton \& Keagan 1988

Botryococcus braunii Kützing 1849

Cadiospora sp.

Calamospora hartungiana Schopf, Wilson \& Bentall 1944

Calamospora microrugosa (Ibrahim) Schopf, Wilson \& Brentall 1944

Calamospora pallida (Loose) Schiopf, Wilson \& Bentall 1944

Calamospora parva Guennel 1958

Convolutispora ampla Hoffmeister, Staplin \& Malloy 1955

Convolutispora insulosa Playford 1978

Cordylosporites magnidictyus (Playford \& Helby) Melo \& Loboziak 2000

Crassispora maculosa (Knox) Sullivan 1964

C. kosankei (Potonié \& Kremp) Bharadwaj 1957 emend. Smith \&

Butterworth 1967

Cristatisporites indignabundus (Potonié \& Kremp) Staplin \& Jansonius 1964

Cristatisporites echinatus Playford 1962

Cristatisporites inordinatus (Menéndez \& Azcuy) Playford 1978

Cyclogranisporites aureus (Loose) Potonié \& Kremp 1955

Cyclogranisporites australis Azcuy 1975

Cyclogranisporites firmus Jones \& Truswell 1992
Cyclogranisporites pisticus Playford 1978

Densosporites anulatus (Loose) Schopf, Wilson \& Bentall 1944

Densosporites pseudoanulatus Butterworth \& Williams 1958

Densosporites stellatus Azcuy 1975

Densosporites variomarginatus Playford 1962

Densosporites sp.

Dictyotriletes sp.

Endoculeospora gradzinskii Turnau 1975

Foveosporites hortonensis (Playford) Azcuy 1975

Foveosporites pellucidus Playford \& Helby 1968

Foveosporites sp.

Granasporites medius (Dybová \& Jachovicz) Ravn,

Butterworth, Phillips \& Peppers 1986

Grandispora daemonii Loboziak et al. 1988

Grandispora debilis Playford 1971

Granulatisporites adnatoides (Potonié \& Kremp) Smith \&

Butterworth 1967

Granulatisporites crenulatus Playford 1964

Granulatisporites frustulentus (Balme \& Hasell) Playford 1971

Granulatisporites parvus (Ibrahim) Schopf, Wilson \& Bentall 1944

Grumosisporites rufus (Butterworth \& Williams) Smith \& Butterworth 1967

Grumosisporites verrucosus (Butterworth \& Williams) Smith \& Butterworth 1967

Grumosisporites varioreticulatus (Neves) Smith \& Butterworth 1967

Knoxisporites literatus (Waltz) Playford 1963

Kraeuselisporites mitratus Higgs 1975

Laevigatosporites sp.

Leiotriletes microgranulatus Playford 1962

Leiotriletes ornatus Ischenko 1956

Leiotriletes sphaerotriangulus (Loose) Potonié \& Kremp 1954

Leiotriletes tenuis Azcuy 1975

Leiotriletes tumidus Butterworth \& Williams 1958

Limitisporites $\mathrm{sp}$.

Potonieisporites sp.

Punctatisporites genuinus Azcuy 1975

Punctatisporites glaber (Naumova) Playford 1962

Punctatisporites humilis Azcuy 1975

Punctatisporites nitidus Hoffmeister, Staplin \& Malloy 1955

Punctatisporites perfrugalis Playford 1978

Punctatisporites cf. resolutus Playford 1971

Punctatisporites trivialis Azcuy 1975

Pustulatisporites sp.

Raistrickia clavata (Hacquebard) Playford 1964

Retusotriletes crassus Clayton in Clayton, Johnston,

Sevastopulo \& Smith 1980

Retusotriletes incohatus Sullivan 1964

Retusotriletes simplex Naumova 1953

Reticulatisporites reticulatus (Ibrahim) Ibrahim 1932

Schopfipollenites ellipsoides (Ibrahim) Potonié \& Kremp 1954

Spelaeotriletes arenaceus Neves \& Owens 1966

Spelaeotriletes triangulus Neves \& Owens 1966

Vallatisporites ciliaris (Luber) Sullivan 1964

Vallatisporites sp.

Velamisporites sp.

Verrucosisporites congestus Playford 1964

Verrucosisporites depressus Winslow 1962

Verrucosisporites gobbettii Playford 1962

Verrucosisporites donarii Potonié \& Kremp 1955

Verrucosisporites minutus Menéndez \& Azcuy 1971

Verrucosisporites morulatus (Knox) Potonié \& Kremp 1955

emend. Smith \& Butterworth 1967

Verrucosisporites nitidus Playford 1964

Waltzispora polita (Hoffmeister et al.) Smith \& Butterworth 1967 